\title{
The effect of an interactive cycling training on cognitive functioning in older adults with mild dementia: study protocol for a randomized controlled trial
}

\author{
E. G. A. Karssemeijer ${ }^{1,2^{*}}$, W. J. R. Bossers ${ }^{3}$, J. A. Aaronson ${ }^{4}$, R. P. C. Kessels ${ }^{4}$ and M. G. M. Olde Rikkert ${ }^{1,2}$
}

\begin{abstract}
Background: To date there is no cure or an effective disease-modifying drug to treat dementia. Available acetylcholine-esterase inhibiting drugs or memantine only produce small benefits on cognitive and behavioural functioning and their clinical relevance remains controversial. Combined cognitive-aerobic interventions are an appealing alternative or add-on to current pharmacological treatments. The primary aim of this study is to investigate the efficacy of a combined cognitive-aerobic training and a single aerobic training compared to an active control group in older adults with mild dementia. We expect to find a beneficial effect on executive functioning in both training regimes, compared to the control intervention, with the largest effect in the combined cognitive-aerobic group. Secondary, intervention effects on cognitive functioning in other domains, physical functioning, physical activity levels, activities of daily living, frailty and quality of life are studied.
\end{abstract}

Methods: The design is a single-blind, randomized controlled trial (RCT) with three groups: a combined cognitiveaerobic bicycle training (interactive cycling), a single aerobic bicycle training and a control intervention, which consists of stretching and toning exercises. Older adults with mild dementia follow a 12-week training program consisting of three training sessions of 30-40 min per week. The primary study outcome is objective executive functioning measured with a neuropsychological assessment. Secondary measures are objective cognitive functioning in other domains, physical functioning, physical activity levels, activities of daily living, frailty, mood and quality of life. The three groups are compared at baseline, after 6 and 12 weeks of training, and at 24-week follow-up.

Discussion: This study will provide novel information on the effects of an interactive cycling training on executive function in older adults with mild dementia. Furthermore, since this study has both a combined cognitive-aerobic training and a single aerobic training group the effectiveness of the different components of the intervention can be identified. The results of this study may be used for physical and mental activity recommendations in older adults with dementia.

Trial registration: The Netherlands National Trial Register NTR5581. Registered 14 February 2016.

Keywords: Dementia, Aerobic training, Combined cognitive-aerobic training, Interactive cycling, Randomized controlled trial, Cognition, Executive functions

\footnotetext{
* Correspondence: Esther.Karssemeijer@radboudumc.nl

'Department of Geriatric Medicine, Donders Institute for Brain Cognition and

Behaviour, Radboud university medical center, Nijmegen, The Netherlands

${ }^{2}$ Radboud university medical center, Radboudumc Alzheimer Center, PO

9101 (hp 925), Nijmegen 6500 HB, The Netherlands

Full list of author information is available at the end of the article
} 


\section{Background}

Dementia is a syndrome characterized by progressive cognitive decline, motor deficits and/or behavioural problems, which increasingly affect the ability to perform activities of daily living [1]. Alzheimer's disease (AD) is the most common cause of dementia accounting for approximately $60-80 \%$ of the dementia cases, followed by vascular dementia [1]. Older age is the strongest risk factor for dementia and due to the aging population the prevalence of dementia is increasing [2]. There are over 9.9 million new cases of dementia each year and the number of persons with dementia is expected to reach 131.50 million in 2050 [3]. Currently, there is no cure or an effective disease-modifying drug to treat dementia [4]. Pharmacological treatment for $\mathrm{AD}$ and vascular dementia with acetylcholinesterase inhibitors (rivastigmine, galantamine, donepezil) or memantine produce small benefits on cognitive and behavioural functioning $[5,6]$. However, the clinical relevance of these pharmacological treatments is controversial and these drugs can cause adverse events (e.g., anorexia, gastrointestinal problems, insomnia) in this vulnerable patient group [6]. Therefore, we should focus on the development and implementation of non-pharmacological interventions as an alternative or add-on therapy. Physical activity seems to be an appealing option [7, 8], as increased lifetime engagement in physical activity reduces the risk of dementia [9] and recent research shows that older adults with dementia spend approximately twothird of the day being sedentary [10].

Recent meta-analyses show positive effects of aerobic exercise interventions on cognitive function in cognitively healthy older adults, with the largest gains in executivecontrol processes [11-14]. Executive function refers to higher-order cognitive processes that controls basic, underlying cognitive functions for non-routine, purposeful, goal-directed behaviour and is linked to prefrontalparietal network activity [15]. Several mechanisms have been identified that may explain this beneficial effect of aerobic exercise on cognitive function: (1) aerobic exercise in aging individuals may increase brain volume, in both grey and white matter, primarily located in the prefrontal and temporal cortices. These brain regions are important for executive control processes and episodic memory, respectively $[16,17] ;(2)$ aerobic exercise may increase the size of the anterior hippocampus, which may lead to improved memory performance [18]; (3) aerobic exercise may enhance neurogenesis in the dentate gyrus of the hippocampus [19]; (4) aerobic exercise may promote extensive cardiovascular changes in the peripheral and cerebral vasculature, such as enhanced angiogenesis [20], and (5) aerobic exercise promotes cardiovascular fitness and therefore reduces peripheral vascular risk factors [21]. Hence, aerobic exercise may have a positive effect on enhancing brain vitality and engagement in physical activity can reduce the risk of dementia-onset in healthy elderly [9].

Several studies have investigated whether physical activity can slow the rate of cognitive decline in older adults with dementia. The results of these studies are mixed. A recently updated meta-analysis of the Cochrane library [22] did not find evidence that physical activity slows cognitive decline in older adults with dementia. In contrast, a meta-analysis of Groot et al. [23] found a beneficial effect of physical activity on cognitive function. This positive effect was independent of the frequency of the intervention and driven by interventions that included aerobic exercise [23]. The opposing outcomes may be explained by the difference in the included studies. Groot et al. incorporated sixteen trials published up to 2015 in the analysis, while the Cochrane library incorporated nine trials and did not include studies after 2013 [22, 23]. Specifically, the most recent studies, reviewed by Groot et al. [23], showed a beneficial effect of physical activity on cognition [24-26]. Moreover, both meta-analyses discuss the large variability in study population, exercise protocols and outcome measures that can complicate interpretation of the results $[22,23]$.

Studies suggest that the neural and cognitive benefits, elicited by physical activity, can be further enhanced if exposure occurs in the context of a cognitively challenging environment [27-29]. Experimental animal studies have shown that physical activity and environmental enrichment (a combination of complex inanimate and social stimulation [30]) differently affect hippocampal neurogenesis, with physical activity influencing the proliferation of neural precursor cells and enriched environment exerting a survival promoting effect on newborn neurons [27, 29]. The findings of previous studies on cognitive effects of single physical training versus combined cognitive-physical training in healthy older adults are in favour of a combined intervention [31, 32]. These combined interventions also seem to positively influence cognition in persons with dementia, with significant effects found on executive function, attention and processing speed [33]. These potential benefits of a combined cognitive-physical training need further investigation since the limited number and heterogeneity of the conducted studies [33]. Moreover, there is a lack of comparison with single physical training interventions to identify the effectiveness of the different components of the intervention. Thus, methodologically high-quality combined cognitive-physical training compared with single physical training studies are needed.

Earlier studies indicate that the gene Apolipoprotein E (APOE) may be involved as a moderator in the effects of physical activity on cognition [34, 35]. APOE is a cholesterol carrier that supports lipid transport and is involved 
in brain injury repair [36]. The APOE gene is polymorphic with three major isoforms: $\varepsilon 2, \varepsilon 3$ and $\varepsilon 4$ [37]. Carrying the $\varepsilon 4$ allele of APOE is the strongest genetic risk factor for developing $\mathrm{AD}$ and carrying the $\varepsilon 2$ allele is protective [2, 38]. Approximately $14 \%$ of the western population carries the $\varepsilon 4$ allele and the estimated prevalence of APOE $\varepsilon 4$ genotype amongst patients diagnosed with $\mathrm{AD}$ is $50 \%[36,39]$. The risk of developing vascular dementia is also elevated in APOE $\varepsilon 4$ carriers, although to a lesser extent [40]. The moderating role of APOE $\varepsilon 4$ in the effect of physical activity on cognition is still unknown. Some epidemiological data suggest that physical activity is more protective in APOE $\varepsilon 4$ carriers compared to non-carriers with respect to incidence of dementia [21]; cerebral amyloid deposition [34]; cognitive function [35, 41]; cognitive decline [42] and memoryrelated brain activation [35]. Other studies, however, suggest that physical activity is related to a lower incidence of dementia and higher level of cognitive functioning in APOE $\varepsilon 4$ non-carriers $[43,44]$. In light of the 'exercise-ismedicine' paradigm, insight in APOE $\varepsilon 4$ moderation may be relevant for the identification of people who will benefit most from physical activity and cognitive stimulation.

The proposed study will expand the scarce research on the cognitive effects of combined cognitive-aerobic training and single aerobic training in older adults with dementia. Furthermore, explorative data will be collected and analyzed to study the moderating effect of APOE status on cognitive and physical function effects.

\section{Objectives and hypothesis}

The primary objective is to study the effect of a 12-week combined cognitive-aerobic bicycle training on executive functioning, compared to a single aerobic bicycle training and an active control group (i.e. stretching and toning), in community-dwelling older adults with mild dementia. We hypothesize that both training regimes will have a positive effect on executive function, compared to the control intervention, with the largest effect in the combined cognitive-aerobic group.

Secondary objectives include investigating i) the effect of training on the cognitive domains of episodic memory, working memory and psychomotor speed, ii) the effect of the training regimes on physical functioning; iii) the effect of training on activities of daily living, mood, quality of life and frailty, and iv) whether the cognitive effects of training are modified by APOE $\varepsilon 4$ carrier state.

\section{Methods \\ Design}

This study is a single-blind, 12-week randomized controlled trial (RCT) with two experimental intervention groups and one active control group. The study design is illustrated in Fig. 1. Participants will be randomly allocated to one of the intervention groups or the active control group. Primary and secondary outcome measures are assessed at baseline and are repeated after the 12 -week intervention period and at 24-weeks in a follow-up assessment. After 6 weeks there is an

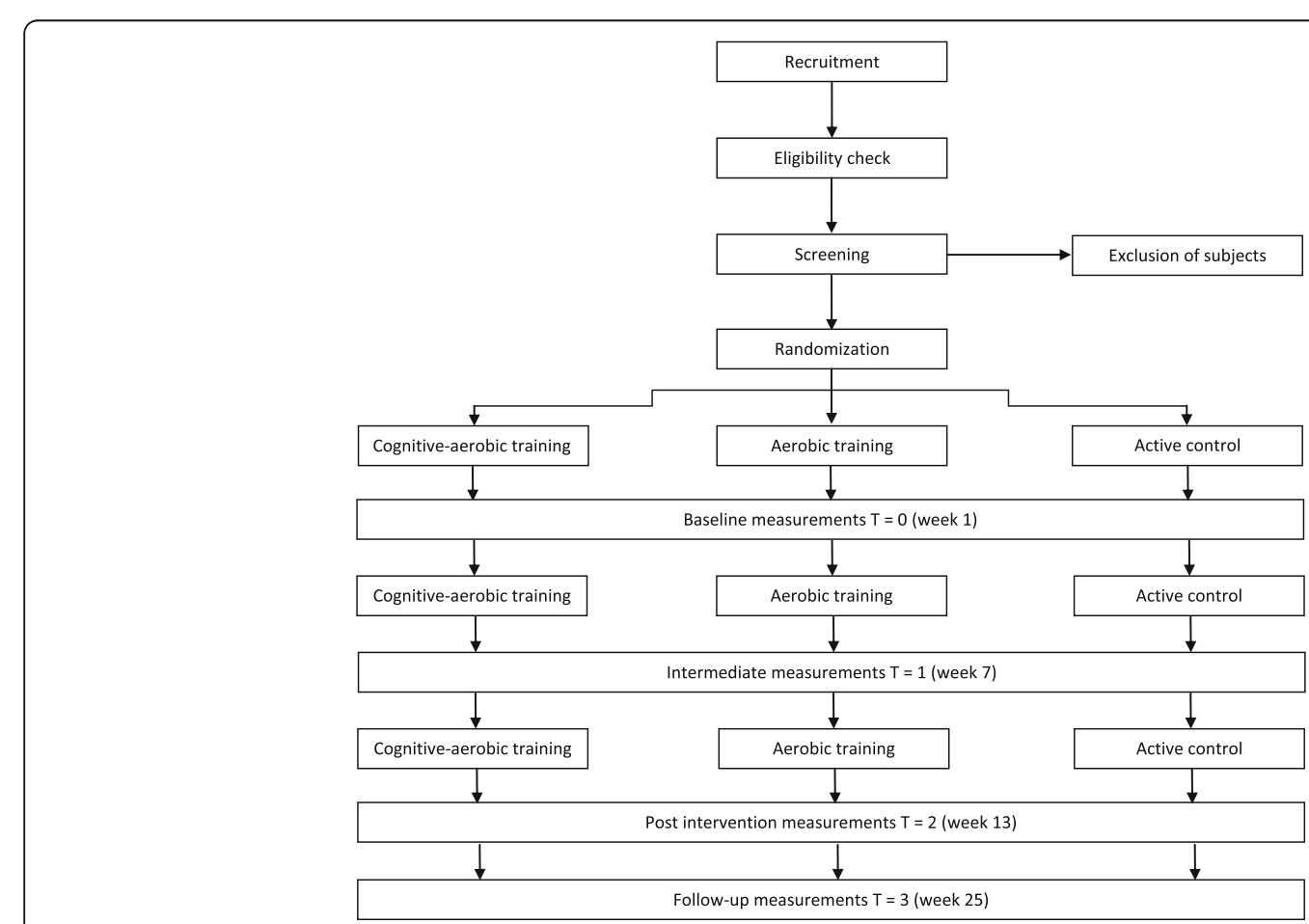

Fig. 1 Flowchart of the study design 
intermediate measurement consisting of the primary outcome measures.

The study protocol has been approved by the Medical Ethical Committee of Radboud university medical center (Ref No: NL52581.091.15/2015-1857) and is registered at the Dutch trial register (http://www.trialregister.nl) with identification number NTR5581. The study is conducted in compliance with Declaration of Helsinki ethical standards.

\section{Patient sample and procedures}

This study is conducted in community centers in the area of Nijmegen, the Netherlands. The study includes persons with a dementia diagnosis (vascular or Alzheimer or mixed type) aged 60 years and older. Exclusion criteria are: moderate or severe dementia defined by a MiniMental Status Examination (MMSE) score of $<17$ [22], incapable to give written consent, comorbidities that limit physical activity (e.g., severe cardiovascular conditions, serious neurological or musculoskeletal problems), diagnosis of a major depression or other psychiatric disorder, drug or alcohol dependency, wheelchair bound and severe hearing or visual problems that cannot be corrected with the use of hearing aids/glasses. Furthermore, participants are excluded if they exercise more than five times per week, at least $30 \mathrm{~min}$ at a moderate intensity [45].

Participants are recruited through the memory clinic of the Radboudumc Alzheimer Center. Potential eligible participants are notified by their physician about the study. Additionally, participants are recruited through day care centres for elderly with cognitive disorders, advertisement in the local newspapers, and word of mouth. All participants who are interested receive detailed information about the nature, purpose and duration of the study, as well as possible objections, risks of participation and the possibility of withdrawal. The researcher contacts the participant to provide further information (if needed) and to invite them to participate. Subsequently, a screening visit is planned. Prior to the screening visit, written informed consent is obtained from the participants. During the screening visit, inclusion and exclusion criteria are assessed. If necessary, permission is asked by the researcher to access hospital files to further evaluate the in- and exclusion criteria. The inclusion period started in February 2016.

\section{Interventions and control condition}

The study includes two intervention groups, which are a combined cognitive-aerobic bicycle training (interactive cycling) and a single aerobic bicycle training group, and an active control condition (stretching and toning). All training groups receive $30-40 \mathrm{~min}$ of training, three times per week for 12 weeks. The training sessions are individually guided by well-trained research assistants.
The trainer records the intensity and duration for each training session. In case of missed training sessions, the reason of absence is recorded. The training sessions are carried out at the participating community centres or day care centres in Nijmegen and the surrounding area.

\section{Aerobic training}

Aerobic training is performed on a stationary bike (Tunturi Go 50 ). Table 1 presents the progressions in intensity and duration during the training period, adapted from the American College of Sports Medicine (ACSM) Guidelines for Exercise Testing and Prescription [46]. Exercise intensity is prescribed using percentage of hearth rate reserve (HRR). Participants on medication that attenuates heart rate (e.g. beta-blockers) are prescribed exercise intensity using the Borg Rating of Perceived Exertion (RPE; [47]). The RPE asks participants to rate their subjective feelings of exertion [47]. Heart rate is monitored with the Polar A300 heart rate monitor. The possibility to increase exercise intensity and duration depends on the individual's physical ability. Duration, intensity and training load are monitored by a trained research assistant.

\section{Interactive cycling training}

The interactive cycling training is a combined cognitiveaerobic bicycle training developed by Fietslabyrint (www.fietslabyrint.nl). Additional file 1 shows the training set-up, in which the home-trainer is connected to a video screen. The aerobic training is identical to the training described above. Additionally, the participants are asked to follow a route through a digital environment presented on the video screen and simultaneously perform cognitive tasks that rely on executive functioning. There are different cognitive training levels and the difficulty of the cognitive tasks increases per level, to ensure that the training remains cognitively challenging. Additional file 2 describes the training tasks in further detail. At the end of each training session participants are provided with feedback on their scores on each task and the scores are registered in a diary. When the participants have a response time of less than $5 \mathrm{~s}$ and an error rate of less than $5 \%$, they can proceed to the next level.

Table 1 Duration and intensity progression of aerobic training

\begin{tabular}{llll}
\hline Week & Duration $(\mathrm{min})$ & Intensity (\% HRR) & Intensity (RPE) \\
\hline $1 \& 2$ & 20 & $50-60$ & $12-15$ \\
$3 \& 4$ & 25 & $50-60$ & $12-15$ \\
$5 \& 6$ & 30 & $60-70$ & $12-15$ \\
$7-9$ & 35 & $60-70$ & $12-15$ \\
$10-12$ & 40 & $65-75$ & $12-15$ \\
\hline
\end{tabular}




\section{Stretching and toning active control group}

Stretching and toning consists of relaxation and flexibility exercises with the same duration and frequency as the other training regimes. The exercises require minimal muscle strength and aerobic capacity and are easy to perform. The level of social engagement is similar to the intervention groups. In persons with dementia, social engagement may have a positive effect on cognitive function [48]. The stretching and toning group thus controls for this social effect. The flexibility exercises consist of upper and lower body exercises, including head rotation, shoulder rotation, shoulders up-down, arm rotation, arm and shoulder muscle strengthening, wrist rotations, flexion/extension fingers, rotation hip, stretching hip flexors and extensors, stretching knee flexors and extensors.

\section{Outcome measures}

\section{Primary outcomes}

The primary outcome measure of this study is objective executive function. Executive function is measured by four neuropsychological tasks, i.e. the short form of the Trail Making Test part B (numbers 1 to 7 and letters A to G) [49]; the abbreviated 5-line Stroop Color Word Test [50, 51]; Letter Fluency [52, 53] and Rule Shift Cards Test [54] [see Additional file 3]. The tests are administered by trained research assistants before and after the training phase (T0 and T2) and at follow up (T3). Parallel versions are used for letter fluency to minimize learning effects at the second and third time point. All the tests, except for letter fluency, are also administered after 6 weeks (T1). The obtained scores are converted into z-scores based on the standard deviation and mean of the total sample at baseline. Subsequently an executive composite z-score is calculated by averaging the z-scores.

\section{Secondary outcomes}

Cognitive measurements: Episodic memory, working memory and psychomotor speed are assessed by neuropsychological assessment. Episodic memory is measured with the Location Learning Test - Revised [55], working memory with the Digit Span subtest (forward and backward condition) from the Wechsler Adult Intelligence Scale - Third Edition (WAIS-III) [56] and the Spatial Span from the Wechsler Memory Scale - Third Edition (WMS-III) [57] and psychomotor speed is assessed using the Trail Making Test part A [49] and the reading and color-naming cards from the abbreviated Stroop ColorWord Test [50]. Additional file 3 describes the cognitive measurements and the scoring methods in more detail. The tests are administered at the same time points as executive function (T0, T2 and T3). A parallel version is used at $\mathrm{T} 2$ for the Location Learning Test to minimize learning effects. The obtained scores are converted into z-scores and a composite z-score is calculated for each domain.

Physical functioning: Physical functioning is measured with performance-based tests suitable for older people. Physical fitness is assessed with the Åstrand Bike Test [58], mobility with the Timed Up \& Go Test [59] and the 10-m Walk Test [60], strength with the 5-times Chair Stand [61] and Handgrip Strength [62] and balance is measured with the Frailty and Injuries Cooperative Studies of Intervention Techniques Subtest 4 [63]. Additional file 4 describes the physical measurements in detail. The different motor domains, strength, physical fitness, balance and mobility, are assessed before and after the training phase (T0 and T2) and at follow-up (T3). At intermediate measurement (T1), only physical fitness and mobility are assessed.

Other secondary outcome measures: Level of physical activity is assessed objectively using an actigraphy device (Philips Actiwatch $2^{\circ}$ ) that participants wear for seven consecutive days and subjectively with the Physical Activity Scale for the Elderly [64]. The Older Persons and Informal Caregivers Survey Minimum DataSet (TOPICS-MDS) [65] is administered to assess activities of daily living and mood. Quality of life is measured with the Dementia Quality of Life Instrument [66] and frailty by using the Evaluative Frailty Index for Physical Activity [67]. These outcome measures are assessed at pre-test (T0) and post-test (T2). Additional file 4 describes the measures in detail.

\section{Moderator}

After inclusion, saliva samples are taken with buccal swabs for APOE genotyping. Buccal samples are stored in $-20{ }^{\circ} \mathrm{C}$ and analyzed using real-time Polymerase Chain Reaction (PCR) [37]. This results in different APOE gene phenotypes: three homozygous $(\varepsilon 2 / \varepsilon 2, \varepsilon 3 / \varepsilon 3$, $\varepsilon 4 / \varepsilon 4)$ and three heterozygous $(\varepsilon 2 / \varepsilon 3, \varepsilon 2 / \varepsilon 4, \varepsilon 3 / \varepsilon 4)$ [37].

\section{Sample size}

Sample size is determined using software package G*power [68]. The effect size (ES) is estimated based on a previous study on the cognitive effects of combined cognitive-aerobic training and single aerobic training using a similar intervention [69]. In this study a medium effect size $(d=0.50)$ was found for executive functioning after 3 months of training. Therefore, assuming a power of 0.80 , an alpha of 0.05 and an expected drop out of $15 \%$, a medium effect size is detected with a total sample size of 171 participants. 
Randomization, blinding and treatment allocation

Participants are randomized after baseline assessments. The minimization technique [70] is used to minimize imbalance between the different groups for gender, severity of cognitive impairment, level of education, use of medication for AD and training location. Minimization is conducted by an independent statistician. Assessors of cognitive outcome measures are blinded to treatment allocation.

\section{Statistical analysis}

Socio-demographic and clinical characteristics at baseline are presented using descriptive statistics. If group differences are observed at baseline, those variables are included as covariates in further analyses. Alpha is set at 0.05 for all analyses. To assess the effect on the primary and secondary outcome measures, analysis of covariance (ANCOVA) is used with cognitive domain scores on the post-tests/intermediate tests as dependent variables, pretest scores as covariates and group (interactive cycling, aerobic bicycle training, control) as between subject factor. In an explorative analysis the moderating effect of APOE $\varepsilon 4$ is evaluated. All analyses are performed as intention-to-treat analysis, including all participants (irrespective of adherence to intervention). Additionally, analyses are rerun as per-protocol analysis. Missing data are substituted using multiple imputation method. Characteristic variables of the sample and cognitive and physical test scores at the different time points will be included in the imputation model. Each imputed dataset will be analysed, pooled and then reported.

\section{Discussion}

Dementia is highly prevalent among older adults. To date no effective disease modifying treatment exists [4]. Combined cognitive-aerobic training seems to be a promising intervention to slow the rate of dementia related cognitive decline. However, up to now, there is insufficient evidence to support its effectiveness. To the best of our knowledge, our study is the first to evaluate the effect of a combined cognitive-aerobic bicycle training and a single aerobic bicycle training on executive functioning in older adults with mild dementia.

One of the major strengths of this study is the design with three groups. Most previous training studies compared a combined cognitive-aerobic training or a single aerobic training with a control group. This study includes both a combined cognitive-aerobic training and a single aerobic training. This gives us the opportunity to assess the differential effects between both training conditions and therewith identify the effectiveness of the different components of the intervention. Another strength is that the difficulty level of the cognitive component in the combined cognitive-aerobic training is adapted to the performance level of the participant. This insures that the training remains cognitively challenging.

A limitation of this study is the relatively short duration of the trial. Previous randomized controlled trials showing cognitive benefits of physical activity or combined cognitive-physical training in older adults with dementia, had intervention periods of 12 weeks or more $[7,33]$. We chose an intervention period of 12 weeks to increase the feasibility and adherence rate and minimize drop-out. Another limitation of this study is that the research population is very heterogeneous as older adults with different types of dementia (Alzheimer, vascular or mixed type) are included. This may affect the internal validity of the study. However, the heterogeneous population will increase the external validity of the results of this study to the community dwelling dementia population.

\section{Conclusions}

The results of this study will provide an important contribution to the existing body of knowledge on combined cognitive-aerobic interventions and single aerobic interventions in older adults with dementia. The results of this study can be important for physical and mental activity recommendations in older adults with dementia.

\section{Additional files}

\author{
Additional file 1: Bicycle set-up. \\ Additional file 2: Description of training levels. \\ Additional file 3: Neuropsychological tests by cognitive domain. \\ Additional file 4: Secondary outcome measurements.
}

\section{Abbreviations}

ACSM: American College of Sports Medicine; AD: Alzheimer's disease; ANCOVA: Analysis of covariance; APOE: Apolipoprotein E; HR: Heart rate; HRR: Heart rate reserve; MMSE: Mini-mental status examination; PCR: Polymerase Chain Reaction; RCT: Randomised controlled trial; RPE: Rate of perceived exertion; TOPICS-MDS: The Older Persons and Informal Caregivers Survey Minimum DataSet; WAIS: Wechsler Adult Intelligence Scale; WMS: Wechsler Memory Scale

\section{Acknowledgments}

This study is part of a collaborative research of a multidisciplinary experts consortium from the University Medical Center Groningen, University of Groningen and VU University Amsterdam, the Netherlands. The aim of the consortium is to determine how specific exercise protocols can counter the negative effects of physical inactivity on dementia progression.

\section{Funding \\ The project is funded by the Netherlands Organisation for Health, Research and Development (ZonMw), grant number 733050303. ZonMw did not contribute to the study design or in writing the manuscript.}

Availability of data and materials

Data sharing not applicable to this article as no datasets were generated or analysed during the current study.

Authors' contributions

MOR and WB developed the idea for the study. EK, WB, JA, RK, MOR contributed to the design of the study. EK coordinates the study under supervision of JA, RK, MOR. EK was the primary author for this manuscript. 
WB and JA helped draft the manuscript. All authors critically reviewed the manuscript and approved for submission.

\section{Competing interests}

The authors declare that they have no competing interests.

\section{Consent for publication}

Not applicable.

\section{Ethics approval and consent to participate}

The study protocol has been approved by the Medical Ethical Committee of Radboud University Medical Center, the Netherlands (Ref No: NL52581.091.15/ 2015-1857). Written informed consent to participate is obtained from all participants.

\section{Publisher's Note}

Springer Nature remains neutral with regard to jurisdictional claims in published maps and institutional affiliations.

\section{Author details}

${ }^{1}$ Department of Geriatric Medicine, Donders Institute for Brain Cognition and Behaviour, Radboud university medical center, Nijmegen, The Netherlands. ${ }^{2}$ Radboud university medical center, Radboudumc Alzheimer Center, PO 9101 (hp 925), Nijmegen 6500 HB, The Netherlands. 'University of Groningen, University Medical Center Groningen, Center for Human Movement Sciences, Groningen, The Netherlands. ${ }^{4}$ Department of Medical Psychology, Donders Institute for Brain Cognition and Behaviour, Radboud university medical center, Nijmegen, The Netherlands.

Received: 27 September 2016 Accepted: 14 March 2017 Published online: 21 March 2017

\section{References}

1. Alzheimer's Association. Alzheimer's disease facts and figures. Alzheimers Dement. 2014;10(3):e47-92

2. Winblad B, Amouyel P, Andrieu S, Ballard C, Brayne C, Brodaty H, et al. Defeating Alzheimer's disease and other dementias: a priority for European science and society. Lancet Neurol. 2016;15(5):455-532.

3. Alzheimer's Disease International: Dementia Statistics. 2015 http://www.alz co.uk/research/statistics. Accessed 30 June 2016

4. Selkoe DJ. Preventing Alzheimer's disease. Science. 2012;337(6101):1488-92.

5. Versijpt J. Effectiveness and cost-effectiveness of the pharmacological treatment of Alzheimer's disease and vascular dementia. J Alzheimers Dis. 2014;42 Suppl 3:19-25

6. Kavirajan H, Schneider LS. Efficacy and adverse effects of cholinesterase inhibitors and memantine in vascular dementia: a meta-analysis of randomised controlled trials. Lancet Neurol. 2007:6(9):782-92.

7. Heyn P, Abreu BC, Ottenbacher KJ. The effects of exercise training on elderly persons with cognitive impairment and dementia: a meta-analysis. Arch Phys Med Rehabil. 2004;85(10):1694-704

8. Deslandes A, Moraes $H$, Ferreira $C$, Veiga $H$, Silveira $H$, Mouta $R$, et al. Exercise and mental health: many reasons to move. Neuropsychobiology. 2009:59(4):191-8.

9. Norton S, Matthews FE, Barnes DE, Yaffe K, Brayne C. Potential for primary prevention of Alzheimer's disease: an analysis of population-based data. Lancet Neurol. 2014;13(8):788-94.

10. van Alphen HJ, Volkers KM, Blankevoort CG, Scherder EJ, Hortobágyi T, van Heuvelen MJ. Older adults with dementia are sedentary for most of the day. PLoS One. 2016;11(3):e0152457

11. Angevaren M, Aufdemkampe $G$, Verhaar $H$, Aleman A, Vanhees L. Physical activity and enhanced fitness to improve cognitive function in older people without known cognitive impairment. Cochrane Database Syst Rev. 2008;3(3):1-73.

12. Voss MW, Nagamatsu LS, Liu-Ambrose T, Kramer AF. Exercise, brain, and cognition across the life span. J Appl Physiol. 2011;111(5):1505-13.

13. Colcombe $\mathrm{S}$, Kramer AF. Fitness effects on the cognitive function of older adults a meta-analytic study. Psychol Sci. 2003:14(2):125-30.

14. Smith PJ, Blumenthal JA, Hoffman BM, Cooper H, Strauman TA, Welsh-Bohmer $K$, et al. Aerobic exercise and neurocognitive performance: a meta-analytic review of randomized controlled trials. Psychosom Med. 2010;72(3):239.
15. Etnier JL, Chang Y-K. The effect of physical activity on executive function: a brief commentary on definitions, measurement issues, and the current state of the literature. J Sport Exerc Psychol. 2009;31:469-83.

16. Colcombe SJ, Erickson KI, Scalf PE, Kim JS, Prakash R, McAuley E, et al. Aerobic exercise training increases brain volume in aging humans. $J$ Gerontol A Biol Sci Med Sci. 2006;61(11):1166-70.

17. Prakash RS, Voss MW, Erickson KI, Kramer AF. Physical activity and cognitive vitality. Annu Rev Psychol. 2015;66:769-97.

18. Erickson Kl, Voss MW, Prakash RS, Basak C, Szabo A, Chaddock L, et al. Exercise training increases size of hippocampus and improves memory. Proc Natl Acad Sci U S A. 2011;108(7):3017-22

19. Nokia MS, Lensu S, Ahtiainen JP, Johansson PP, Koch LG, Britton SL, et al. Physical exercise increases adult hippocampal neurogenesis in male rats provided it is aerobic and sustained. J Physiol. 2016;594(7):1855-73.

20. Lange-Asschenfeldt C, Kojda G. Alzheimer's disease, cerebrovascular dysfunction and the benefits of exercise: from vessels to neurons. Exp Gerontol. 2008:43(6):499-504

21. Rovio S, Kåreholt I, Helkala E-L, Viitanen M, Winblad B, Tuomilehto J, et al. Leisure-time physical activity at midlife and the risk of dementia and Alzheimer's disease. Lancet Neurol. 2005;4(11):705-11.

22. Forbes D, Forbes SC, Blake CM, Thiessen EJ, Forbes S. Exercise programs for people with dementia. Cochrane Database Syst Rev. 2015;5:1-18.

23. Groot $C$, Hooghiemstra A, Raijmakers $P$, van Berckel B, Scheltens $P$, Scherder $E$, et al. The effect of physical activity on cognitive function in patients with dementia: a meta-analysis of randomized control trials. Ageing Res Rev. 2016;25:13-23

24. Bossers WJ, van der Woude LH, Boersma F, Hortobágyi T, Scherder EJ, van Heuvelen MJ. A 9-week aerobic and strength training program improves cognitive and motor function in patients with dementia: a randomized, controlled trial. Am J Geriatr Psychiatry. 2015;23(11):1106-16.

25. Arcoverde C, Deslandes A, Moraes H, Almeida C, Araujo NB, Vasques PE, et al. Treadmill training as an augmentation treatment for Alzheimer's disease: a pilot randomized controlled study. Arq Neuropsiquiatr. 2014;72(3):190-6.

26. Cheng S-T, Chow PK, Song Y-Q, Edwin C, Lam JH. Can leisure activities slow dementia progression in nursing home residents? A cluster-randomized controlled trial. Int Psychogeriatr. 2014;26(04):637-43.

27. Fabel K, Wolf SA, Ehninger D, Babu H, Leal-Galicia P, Kempermann G. Additive effects of physical exercise and environmental enrichment on adult hippocampal neurogenesis in mice. Front Neurosci. 2009;3:50.

28. Fabel K, Kempermann G. Physical activity and the regulation of neurogenesis in the adult and aging brain. Neruomol Med. 2008;10(2):59-66.

29. Olson AK, Eadie BD, Ernst C, Christie BR. Environmental enrichment and voluntary exercise massively increase neurogenesis in the adult hippocampus via dissociable pathways. Hippocampus. 2006;16(3):250-60.

30. Rosenzweig MR, Bennett EL, Hebert M, Morimoto H. Social grouping cannot account for cerebral effects of enriched environments. Brain Res. 1978; 153(3):563-76.

31. Oswald WD, Gunzelmann T, Rupprecht R, Hagen B. Differential effects of single versus combined cognitive and physical training with older adults: the SimA study in a 5-year perspective. Eur J Ageing. 2006;3(4):179-92.

32. Fabre C, Chamari K, Mucci P, Masse-Biron J, Prefaut C. Improvement of cognitive function by mental and/or individualized aerobic training in healthy elderly subjects. Int J Sports Med. 2002;23(6):415-21.

33. Law LL, Barnett F, Yau MK, Gray MA. Effects of combined cognitive and exercise interventions on cognition in older adults with and without cognitive impairment: a systematic review. Ageing Res Rev. 2014;15:61-75.

34. Head D, Bugg JM, Goate AM, Fagan AM, Mintun MA, Benzinger T, et al. Exercise engagement as a moderator of the effects of APOE genotype on amyloid deposition. Arch Neurol. 2012;69(5):636-43.

35. Deeny SP, Poeppel D, Zimmerman JB, Roth SM, Brandauer J, Witkowski S, et al. Exercise, APOE, and working memory: MEG and behavioral evidence for benefit of exercise in epsilon4 carriers. Biol Psychol. 2008:78(2):179-87.

36. Liu C-C, Kanekiyo T, Xu H, Bu G. Apolipoprotein E and Alzheimer disease: risk, mechanisms and therapy. Nat Rev Neurol. 2013;9(2):106-18.

37. Yi L, Wu T, Luo W, Zhou W, Wu J. A non-invasive, rapid method to genotype late-onset Alzheimer's disease-related apolipoprotein E gene polymorphisms. Neural Regen Res. 2014;9(1):69.

38. Farrer LA, Cupples LA, Haines JL, Hyman B, Kukull WA, Mayeux R, et al. Effects of age, sex, and ethnicity on the association between apolipoprotein E genotype and Alzheimer disease: a meta-analysis. JAMA. 1997:278(16): $1349-56$ 
39. Ward A, Crean S, Mercaldi CJ, Collins JM, Boyd D, Cook MN, et al. Prevalence of apolipoprotein E4 genotype and homozygotes (APOE e4/4) among patients diagnosed with Alzheimer's disease: a systematic review and meta-analysis. Neuroepidemiology. 2011;38(1):1-17.

40. Rohn TT. Is apolipoprotein E4 an important risk factor for vascular dementia? Int J Clin Exp Pathol. 2014;7(7):3504.

41. Etnier JL, Caselli RJ, Reiman EM, Alexander GE, Sibley BA, Tessier D, et al. Cognitive performance in older women relative to ApoE-e4 genotype and aerobic fitness. Med Sci Sports Exerc. 2007;39(1):199-207.

42. Schuit AJ, Feskens E, Launer $\amalg$, Kromhout D. Physical activity and cognitive decline, the role of the apolipoprotein e4 allele. Med Sci Sports Exerc. 2001; 33(5):772-7.

43. Podewils LJ, Guallar E, Kuller LH, Fried LP, Lopez OL, Carlson M, et al. Physical activity, APOE genotype, and dementia risk: findings from the Cardiovascular Health Cognition Study. Am J Epidemiol. 2005;161(7):639-51.

44. Obisesan TO, Umar N, Paluvoi N, Gillum RF. Association of leisure-time physical activity with cognition by apolipoprotein-E genotype in persons aged 60 years and over: the National Health and Nutrition Examination Survey (NHANES-III). Clin Interv Aging. 2012;7:35.

45. Nelson ME, Rejeski WJ, Blair SN, Duncan PW, Judge JO, King AC, et al. Physical activity and public health in older adults: recommendation from the American College of Sports Medicine and the American Heart Association. Circulation. 2007;116(9):1094.

46. American College of Sports Medicine. Exercise Prescription In: ACSM's guidelines for exercise testing and prescription. Franklin BA, editor. Baltimore: Lippincott Williams \& Wilkins; 2000. 135-234.

47. Borg G. Borg's perceived exertion and pain scales. Hum Kinet. 1998.

48. Bassuk SS, Glass TA, Berkman LF. Social disengagement and incident cognitive decline in community-dwelling elderly persons. Ann Intern Med. 1999:131(3):165-73.

49. Reitan RM, Wolfson D. The Trail Making Test as an initial screening procedure for neuropsychological impairment in older children. Arch Clin Neuropsychol. 2004;19(2):281-8.

50. Jensen AR, Rohwer WD. The Stroop color-word test: A review. Acta Psychol. 1966;25:36-93

51. Yogev G, Giladi N, Peretz C, Springer S, Simon ES, Hausdorff JM. Dual tasking, gait rhythmicity, and Parkinson's disease: which aspects of gait are attention demanding? Eur J Neurosci. 2005;22(5):1248-56.

52. Ruff R, Light R, Parker S, Levin H. Benton controlled oral word association test: Reliability and updated norms. Arch Clin Neuropsychol. 1996;11(4):329-38.

53. Schmand B, Groenink S, Van den Dungen M. Letter fluency: psychometric properties and Dutch normative data. Tijdschr Gerontol Geriatr. 2008;39(2):64-76.

54. Wilson BA, Evans JJ, Alderman N, Burgess PW, Emslie H. Behavioural assessment of the dysexecutive syndrome. In: Rabbit $P$, editor. Methodology of frontal and executive function. 1997. p. 239-50.

55. Bucks R, Willison J, Byrne L, Kessels R. Location learning test-revised edition (Dutch version). 2011

56. Wechsler D. Wechsler adult intelligence scale 3rd edition (WAIS-III): Test manual. New York: NY Psychological Corporation; 1997.

57. Wechsler D. Wechsler memory scale (WMS-III): Administration and Scoring Manual. San Antonio: TX Psychological Corporation; 1997.

58. Åstrand P-O, Ryhming I. A nomogram for calculation of aerobic capacity (physical fitness) from pulse rate during submaximal work. J Appl Physiol. 1954;7(2):218-21.

59. Podsiadlo D, Richardson S. The timed "Up \& Go": a test of basic functional mobility for frail elderly persons. J Am Geriatr Soc. 1991;39(2):142-8.

60. Wade DT. Measurement in neurological rehabilitation. Curr Opin Neurol. 1992;5(5):682-6.

61. Bohannon RW. Sit-to-stand test for measuring performance of lower extremity muscles. Percept Mot Skills. 1995;80(1):163-6.

62. Rantanen T, Guralnik JM, Foley D, Masaki K, Leveille S, Curb JD, et al. Midlife hand grip strength as a predictor of old age disability. JAMA. 1999;281(6):558-60

63. Ory MG, Schechtman KB, Miller JP, Hadley EC, Fiatarone MA, Province MA, et al. Frailty and injuries in later life: the FICSIT trials. J Am Geriatr Soc. 1993;41(3):283-96.

64. Washburn RA, Smith KW, Jette AM, Janney CA. The Physical Activity Scale for the Elderly (PASE): development and evaluation. J Clin Epidemiol. 1993; 46(2):153-62.

65. Lutomski JE, Baars MA, Schalk BW, Boter H, Buurman BM, den Elzen WP, et al. The development of the Older Persons and Informal Caregivers Survey Minimum DataSet (TOPICS-MDS): a large-scale data sharing initiative. PLoS One. 2013;8(12):e81673.
66. Brod M, Stewart AL, Sands L, Walton P. Conceptualization and measurement of quality of life in dementia: The dementia quality of life instrument (DQoL). Gerontologist. 1999;39(1):25-36.

67. de Vries NM, Staal JB, Rikkert MGO. Nijhuis-van der Sanden MW. Evaluative frailty index for physical activity (EFIP): a reliable and valid instrument to measure changes in level of frailty. Phys Ther. 2012;93:551-61.

68. Erdfelder E, Faul F, Buchner A. GPOWER: A general power analysis program. Behav Res Methods Instrum Comput. 1996;28(1):1-11.

69. Anderson-Hanley C, Arciero PJ, Brickman AM, Nimon JP, Okuma N, Westen SC, et al. Exergaming and older adult cognition: a cluster randomized clinical trial. Am J Prev Med. 2012:42(2):109-19.

70. Pocock SJ, Simon R. Sequential treatment assignment with balancing for prognostic factors in the controlled clinical trial. Biometrics. 1975;31:103-15.

\section{Submit your next manuscript to BioMed Central and we will help you at every step:}

- We accept pre-submission inquiries

- Our selector tool helps you to find the most relevant journal

- We provide round the clock customer support

- Convenient online submission

- Thorough peer review

- Inclusion in PubMed and all major indexing services

- Maximum visibility for your research

Submit your manuscript at www.biomedcentral.com/submit
) Biomed Central 